\title{
COMPARAÇÃO ENTRE DIFERENTES MÉTODOS DE ESTIMATIVA DE EVAPOTRANSPIRAÇÃO DE REFERÊNCIA (ETo) PARA LINHARES, ES
}

\author{
GARDIMAN JUNIOR, Benvindo Sirtoli ${ }^{1}$ \\ MAGALHÃES, Ivo Augusto Lopes ${ }^{2}$ \\ CECILIO, Roberto Avelino ${ }^{3}$
}

\begin{abstract}
RESUMO: Este trabalho teve como objetivo comparar o desempenho de equações empíricas de Thornthwaite, Camargo, Camargo Simplificado e Garcia Lopez e física de Priestley Taylor, para estimativa da evapotranspiração de referência, comparando-as ao método universal de Penman - Montheit FAO (1998) para as condições climáticas da localidade de Linhares, no litoral norte do Estado do Espírito Santo. Foram utilizadas as variáveis climáticas de temperaturas médias do $\operatorname{ar}\left({ }^{\circ} \mathrm{C}\right)$, umidade relativa $(\%)$ e radiação global $\left(\mathrm{MJ} \mathrm{m}^{-2}\right)$ referentes ao período de 10 meses (janeiro a outubro de 2008), adquiridas pela plataforma de dados do CEUNES - UFES, provenientes da estação automatizada meteorológica, código n. A614, instalada no município citado a 40 metros acima do nível do mar. Para comparar os valores de ETo estimados por meio das equações empíricas e física com os métodos de Penman Montheit FAO (1998), foram considerados os parâmetros da equação de regressão $\beta 0$ e $\beta 1$, modelagem da eficiência $(\mathrm{EF} \%)$, coeficiente de determinação $\left(\mathrm{r}^{2}\right)$, coeficiente de correlação (r), estimativa do erro padrão (EEP), índice de concordância (d), índice de confiança ou desempenho (c), para as escalas diária, acumulado de três, sete dias e mensal, para os meses estudados em ambos os países. Para as condições climáticas de onde se realizou o estudo, em relação aos índices, parâmetros estudados e escalas de tempo, o método com desempenho superior foi o de Garcia Lopez.
\end{abstract}

Palavras-chave: Equações empíricas. Evapotranspiração de referência. Métodos de estimação.

\section{COMPARISON BETWEEN DIFFERENT METHODS OF ESTIMATING REFERENCE EVAPOTRANSPIRATION (ETo) FOR LINHARES, ES}

SUMMARY: The aim of this study is to compare the performance of empirical equations of Thornthwaite, Camargo, Camargo and Garcia Lopez Simplified physical Priestley and Taylor, to estimate the evapotranspiration reference, comparing them to the universal Penman's method - Montheit FAO (1998) to conditions weather in the city of Linhares, in the north coast of Espírito Santo. That was used the climatic variables of average air temperatures $\left({ }^{\circ} \mathrm{C}\right)$, humidity (\%) and global radiation $\left(\mathrm{MJ}^{-\mathrm{m}^{-2}}\right.$ ) during 10 months (January to December of 2008) acquired by the platform's data CEUNES - UFES from automated weather station, code n. A614, in the same city said before, installed the 40 meters at sea level. To compare the ETo values estimated by the empirical equations and physics with the Penman's methods of - Montheit FAO 1998 were considered the parameters of the regression equation $\beta 0$ and $\beta 1$, efficiency model (EF\%), determination's coefficient $\left(\mathrm{r}^{2}\right)$, correlation's coefficient ( $\mathrm{r}$ ), standard error estimated (SEE), index's agreement (d), confidence's index or performance (c) for the daily scales, cumulative three, seven days and monthly for the studied months in both countries. For the weather conditions where the study took place and was compared to the indexs, sturdy's parameters and time scales, the method with the best performance was from Garcia Lopez.

Keywords: Empirical equations. Reference evapotranspiration. Estimation methods.

\section{INTRODUÇÃO}

Água e solo são recursos fundamentais do ambiente natural para atividades agrícolas e sua disponibilidade é essencial para o crescimento e desenvolvimento dos vegetais. No que se refere à

\footnotetext{
${ }^{1}$ Engenheiro Ambiental, Mestrando em Ciências Florestais

2 Engenheiro Ambiental. Mestre em Ciências Florestais/Sensoriamento remoto e manejo de bacias hidrográficas. - UFES

${ }^{3}$ Dr. Prof. em Engenharia Agrícola-UFES
} 
quantidade e qualidade, os recursos hídricos estão cada vez mais escassos, tanto para o consumo humano como para a produção agrícola. Para tanto, torna-se necessário um planejamento mais eficaz do aproveitamento da água, com desenvolvimento de metodologias que permitam estimar volumes cada vez mais exatos para obtenção de ótima produção nos cultivos agrícolas. A disponibilidade de água do solo para as plantas não depende apenas das precipitações pluviais, mas também da outra componente do balanço hídrico que corresponde às perdas de água do solo para a atmosfera, que ocorrem por evaporação e transpiração vegetal, processo denominado de evapotranspiração (NERY; CASTAÑEDA, 1992). Tal termo foi utilizado por Thornthwaite, no início da década de 40 para expressar a ocorrência simultânea dos processos de evaporação e transpiração em uma superfície vegetada (PEREIRA et al., 1997).

Este processo físico de mudança de estado da água pode ser influenciado por alguns fatores climáticos e controlado pela disponibilidade de energia, pela demanda atmosférica por vapor de água e pelo suprimento de água do solo às plantas. A espécie cultivada e seu estádio de desenvolvimento são fatores da própria planta que também podem influenciar na evapotranspiração (ALLEN et al., 1998; PEREIRA et al., 2002).

A evapotranspiração de referência (ETo), segundo Doorenbos e Pruitt (1997) e Allen et al. (1998), é aquela que ocorre em uma extensa superfície de grama com altura de 0,08 $\mathrm{m}$ a 0,15 m, em crescimento ativo, cobrindo totalmente o solo e sem deficiência de água.

Apesar da existência de diversos modelos para se estimar a ETo, no entanto, tais estimadores são utilizados em condições climáticas e agronômicas muito diferentes daquelas em que inicialmente foram concebidos e, por isso, é de extrema importância avaliar o grau de exatidão desses modelos, antes de utilizá-los para nova condição (OLIVEIRA, 2001).

Allen et al. (1998) citam que, consultores da Organização das Nações Unidas para Alimentação e Agricultura (FAO), revisando os métodos de estimativa da ETo, deliberaram que a equação de PenmanMonteith deve ser utilizada como método padrão, porque é baseada em processos físicos e incorpora parâmetros fisiológicos e aerodinâmicos.

Camargo e Camargo (2000), completam que a equação de Penman-Monteith prediz com eficácia a ETo em diversas condições de umidade atmosférica, necessitando de vários elementos meteorológicos que nem sempre se encontram disponíveis em algumas regiões. Para isso a alternativa é o uso de equações empíricas que considerem a ETo um elemento meteorológico padrão para emprego na agrometeorologia.

Dentre os métodos de estimativa, os denominados empíricos, têm aplicabilidade quase universal e resultam de correlações entre os elementos meteorológicos medidos em condições padrões, além da evapotranspiração que também é medida em condições padronizadas. Os métodos combinados reúnem os efeitos do balanço de energia àqueles do poder evaporante do ar (PEREIRA et al., 2002).

Para Syperreck et al. (2008), o desempenho destes métodos na estimativa da evapotranspiração de referência pode ser determinado por meio dos índices que determinarão a precisão e exatidão. Estes índices também têm sido aplicados em diversos outros trabalhos (ARAÚJO et al., 2007; REIS et al., 2007; OLIVEIRA et al., 2008; PEREIRA et al., 2009; OLIVEIRA et al., 2010; ALENCAR et al., 2011; SILVA et al., 2011) de forma a comparar valores estimados aos observados com bons resultados.

Para o Estado do Espírito Santo, a quantificação da evapotranspiração assume particular importância em virtude dos déficits hídricos ao longo do ano (SCÁRDUA et al., 1986), por possuir extensas áreas de agricultura irrigada na região norte do estado, objeto deste estudo.

Diante do contexto apresentado, este trabalho teve como objetivo avaliar o desempenho dos métodos empíricos de Thornthwaite, Camargo, Camargo Simplificado e Garcia Lopez e método físico de Priestley-Taylor, comparando-os ao método padrão de Penman - Montheit FAO (1998) e para a localidade de Linhares, estado do Espírito Santo.

Nucleus, v.9, n.2, out.2012 


\section{MATERIAL E MÉTODOS}

Este trabalho foi realizado com o auxílio dos dados meteorológicos coletados na Estação Meteorológica Automática do Centro Universitário Norte do Espírito Santo (CEUNES) da Universidade Federal do Espírito Santo (UFES) para a cidade de Linhares (A614), compreendida na coordenada Latitude $19^{\circ} 21^{\prime} \mathrm{S}$ e Longitude $40^{\circ} 04^{\prime} \mathrm{W}$ a uma altitude média de 40 metros, ou seja, região litoral norte, conforme representado na Figura 1.

Figura 1 - Localização da estação meteorológica automática de Linhares, ES.

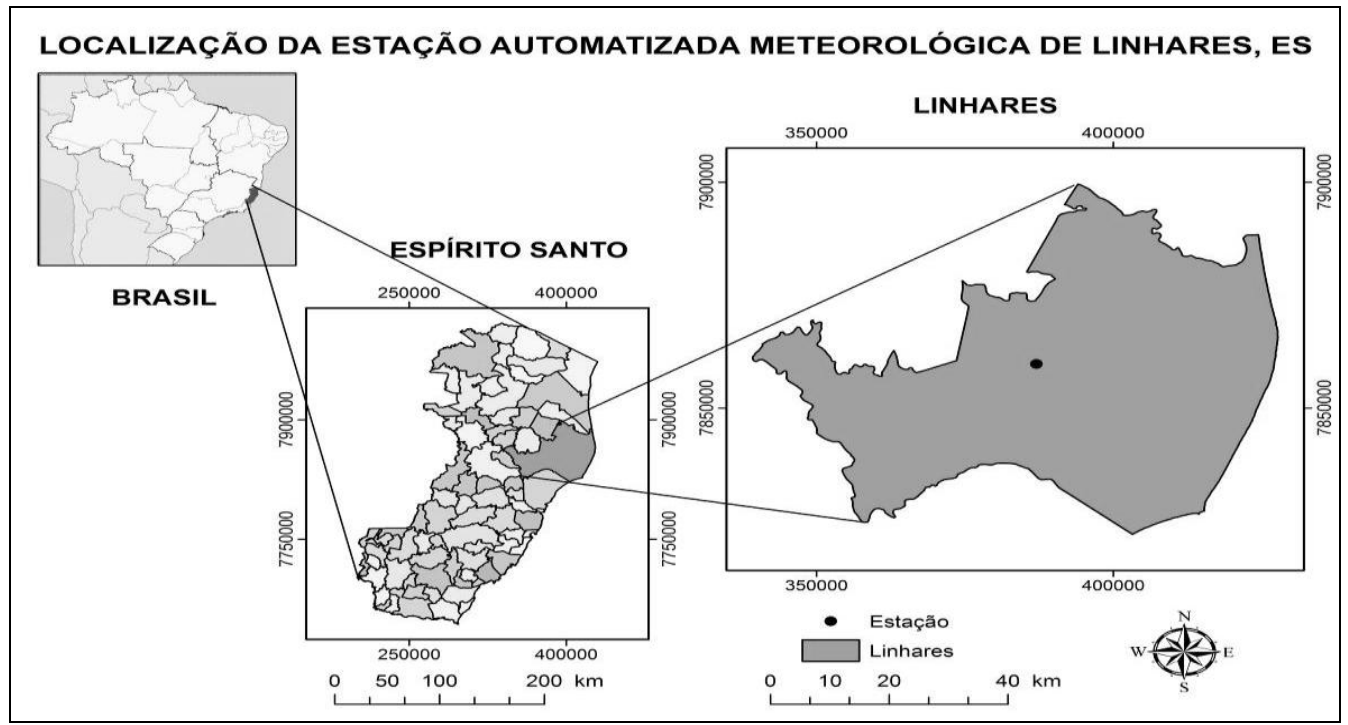

O clima da região de Linhares segundo classificação de Köppen é Aw, tratando-se de clima tropical úmido com inverno seco. As médias anuais da precipitação giram em torno dos $1.277 \mathrm{~mm}$, enquanto que a evapotranspiração potencial anual média é de cerca $1.224 \mathrm{~mm}$ e a deficiência hídrica aproximadamente $69 \mathrm{~mm}$ (NÓBREGA et al., 2006).

Foram obtidos dados do período entre janeiro a outubro de 2008, de temperaturas médias do ar $\left({ }^{\circ} \mathrm{C}\right)$, umidade relativa $(\%)$ e radiação global $\left(\mathrm{MJ} \mathrm{m}^{-2}\right)$ das referidas estações. Os parâmetros "I", índice que expressa o nível de calor disponível na região em ${ }^{\circ} \mathrm{C}$ e o expoente "a" para o método de Thornthwaite foram obtidos nas normais climatológicas, uma vez que são característicos da região, tornam-se constantes e independentes do ano de estimativa.

O fator de correção (Cor) da evapotranspiração em função do fotoperíodo e do número de dias do mês para o método simplificado de Camargo (1962) foi obtido na tabela de Camargo (1964), assim como também a evapotranspiração potencial diária $\left(\mathrm{ET}_{\mathrm{T}} \mathrm{mm}\right.$ dia $\left.^{-1}\right)$ em função das temperaturas médias anual (Ta) e mensal (Tm) ou diária (Td) foram obtidas da tabela de Camargo (1962) apresentado por Pereira et al. (2002).

De posse dos valores médios das variáveis climáticas, a evapotranspiração de referência (ETo) foi estimada para o período diário, acumulado de três e sete dias e mensal pelos métodos do Thornthwaite (1948), Penman-Monteith FAO (1998), simplificação de Camargo (1962), Camargo (1971), Priestley e Taylor (1972) e Garcia Lopez (1970).

Posteriormente, cada valor de ETo foi correlacionado com o valor correspondente ao mesmo período estimado pelo método Penman-Monteith FAO (1998), considerado padrão, obtendo-se a precisão dos dados através do coeficiente de determinação $\left(\mathrm{r}^{2}\right)$, o qual indica o grau em que a regressão explica a soma do quadrado total. Além disso, comparou-se o coeficiente de correlação (r), parâmetros da equação 
de regressão ( $\beta 0$ e $\beta 1)$ e teste de significância a 5\% realizado no programa computacional livre IRENE 1.0 .

Calculou-se o índice de concordância (d) proposto por Willmont et al. (1985) conforme Equação 1 onde seus valores variam de zero (não existe concordância) a "um" (concordância perfeita).

$$
d=1-\frac{\sum_{i=1}^{n}(P i-O i)^{2}}{\sum_{i=1}^{n} \mathbf{P} i-\bar{o}+Q_{i-\bar{o}}^{\overline{2}}}
$$

em que,

d é o índice de concordância ou ajuste;

Pi é a evapotranspiração de referência obtida pelo método considerado, $\mathrm{mm} \mathrm{dia}^{-{ }^{-1}}$;

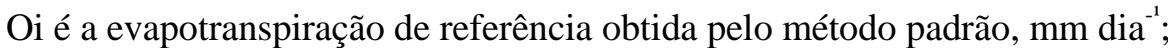

O é a média dos valores de ETo obtida pelo método padrão, $\mathrm{mm} \mathrm{dia}^{-1}, \mathrm{e}$;

n é o número de observações.

A estimativa do erro padrão (EEP) proposta por Allen et al. (1986) (Equação 2), onde quanto maior o valor, mais subestima a ETo, usada para comparar os resultados juntamente com a modelagem do percentual de eficiência (EF\%) proposta por Greenwood et al. (1985), onde maior, mais distante do valor comparado (Equação 3).

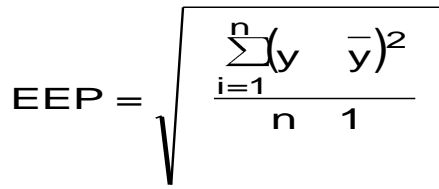

onde,

EEP é a estimativa do erro padrão, $\mathrm{mm} \mathrm{dia}^{-{ }^{-1}}$;

y é a evapotranspiração de referência estimada pelo método padrão, $\mathrm{mm} \mathrm{dia}^{-1}$;

$\bar{y}$ é a evapotranspiração de referência obtida pelo considerado, $\mathrm{mm}$ dia $^{-1}, \mathrm{e}$;

n é o número de observações.

$$
F \%=100 \cdot\left(\frac{\sum_{i=1}^{n} \boldsymbol{E}_{i}-M_{i}^{\mathbf{z}}}{\sum_{i=1}^{n} \boldsymbol{E}_{i}-\bar{M}_{i}^{\boldsymbol{\nu}} \boldsymbol{\nu}}\right)
$$

em que,

EF\% é a modelagem do percentual de eficiência;

Ei é a evapotranspiração de referência estimada pelo método considerado, $\mathrm{mm} \mathrm{dia}^{-1}$;

Mi é a evapotranspiração de referência obtida pelo método padrão, mm. dia ${ }^{-1}$; e;

$\bar{M}$ i é a evapotranspiração média de referência obtida pelo método padrão, mm. dia ${ }^{-1}$;

Utilizou-se o índice de confiança ou desempenho (c), proposto por Camargo e Sentelhas (1996), que serve como indicador de desempenho dos métodos. É representado pelo produto dos índices de precisão "r" e de exatidão "d”, expresso conforme Equação 4.

$$
c=r * d
$$

Os valores de "c" são interpretados de acordo com os referidos autores como: "ótimo" $(c>0,85)$; "muito bom" ( c entre 0,76 e 0,85); "bom" (c entre 0,66 e 0,75); "mediano" ( c entre 0,61 e 0,65), "sofrível" (c entre 0,51 e 0,60), "mau" (c entre 0,41 e 0,50) e "péssimo" (c < 0,40). 


\section{RESULTADOS E DISCUSSÃO}

Na Tabela 1 encontram-se os resultados dos métodos de evapotranspiração estudados nas escalas diária, acumulado de três, sete dias e mensal para a localidade de Linhares, juntamente com os parâmetros analisados.

Tabela 1. Parâmetros da equação $(\beta 0, \beta 1)$, modelagem do percentual de eficiência (EF\%), coeficiente de determinação $\left(\mathrm{r}^{2}\right)$, estimativa do erro padrão (EEP), coeficiente de correlação(r), índice de concordância (d), índice de confiança ou desempenho (c), classificação e valores da ET0 diárias, acumulado de três, sete dias e mensal, calculados para o período de janeiro a outubro de 2008 na localidade de Linhares-ES

\begin{tabular}{|c|c|c|c|c|c|c|c|c|c|c|c|}
\hline Métodos & $\beta 0$ & $\beta 1$ & $\mathrm{EF} \%$ & $\mathrm{Th}$ & $\mathrm{r}^{2}$ & EEP & $\mathrm{r}$ & $\mathrm{d}$ & $\mathrm{c}$ & Classificação & ETo \\
\hline \multicolumn{12}{|c|}{ Diária } \\
\hline $\begin{array}{l}\text { Penman Monteith } \\
\text { FAO (1998) }\end{array}$ & & & & & & & & & & & 4,1 \\
\hline Priestley Taylor & $-1,100$ & 2,056 & 1115,58 & $* *$ & 14,20 & 3,529 & 0,97 & 0,499 & 0,48 & Mau & 7,4 \\
\hline Thornthwait & 3,670 & 0,155 & 79,83 & $*$ & 0,09 & 0,944 & 0,59 & 0,471 & 0,28 & Péssimo & 4,3 \\
\hline Camargo Simplificado & 0,808 & 0,570 & 129,16 & $* *$ & 1,22 & 1,201 & 0,59 & 0,612 & 0,36 & Péssimo & 3,3 \\
\hline Camargo & 0,019 & 1,052 & 167,29 & $* *$ & 1,39 & 1,366 & 0,50 & 0,542 & 0,75 & Bom & 3,1 \\
\hline Garcia Lopes & $-0,816$ & 0,950 & 44,35 & $*$ & 0,56 & 0,704 & 0,75 & 0,839 & 0,63 & Mediano & 4,2 \\
\hline \multicolumn{12}{|c|}{ Acumulado de 3 dias } \\
\hline $\begin{array}{l}\text { Penman Monteith } \\
\text { FAO (1998) }\end{array}$ & & & & & & & & & & & 10,2 \\
\hline Priestley Taylor & 2,824 & 2,066 & 602,36 & $* *$ & 8,23 & 7,781 & 0,87 & 0,543 & 0,47 & Mau & 17,3 \\
\hline Thornthwait & 11,512 & 0,136 & 151,23 & $* *$ & 0,55 & 3,899 & 0,73 & 0,555 & 0,40 & Mau & 12,9 \\
\hline Camargo Simplificado & 2,916 & 0,596 & 44,58 & * & 0,75 & 2,117 & 0,81 & 0,859 & 0,70 & Bom & 9,1 \\
\hline Camargo & 3,251 & 0,569 & 44,94 & $*$ & 0,67 & 2,125 & 0,81 & 0,850 & 0,69 & Bom & 9,1 \\
\hline Garcia Lopes & 5,780 & 0,514 & 44,12 & * & 0,48 & 2,106 & 0,81 & 0,834 & 0,68 & Bom & 11 \\
\hline \multicolumn{12}{|c|}{ Acumulado de 7 dias } \\
\hline $\begin{array}{l}\text { Penman Monteith } \\
\text { FAO (1998) }\end{array}$ & & & & & & & & & & & 29 \\
\hline Priestley Taylor & $\begin{array}{c}- \\
12,668\end{array}$ & 2,228 & 4040,29 & $* *$ & 43,72 & 24,357 & 0,92 & 0,259 & 0,24 & Péssimo & 52,7 \\
\hline Thornthwait & 20,584 & 0,329 & 63,08 & $*$ & 0,28 & 3,044 & 0,74 & 0,695 & 0,52 & Sofrível & 30,2 \\
\hline Camargo Simplificado & $-7,912$ & 1,042 & 366,99 & $* *$ & 4,57 & 7,341 & 0,62 & 0,582 & 0,36 & Péssimo & 23,3 \\
\hline Camargo & $\begin{array}{c}- \\
11,869\end{array}$ & 1,139 & 429,37 & $* *$ & 5,38 & 7,940 & 0,71 & 0,559 & 0,40 & Péssimo & 22,2 \\
\hline Garcia Lopes & 0,373 & 0,972 & 51,00 & $*$ & 1,39 & 2,737 & 0,80 & 0,886 & 0,71 & Bom & 28,9 \\
\hline \multicolumn{12}{|c|}{ Acumulado Mensal } \\
\hline $\begin{array}{l}\text { Penman Monteith } \\
\text { FAO (1998) }\end{array}$ & & & & & & & & & & & 116,2 \\
\hline Priestley Taylor & 13,125 & 1,618 & 3108,67 & $* *$ & 33,31 & 86,184 & 0,93 & 0,285 & 0,27 & Péssimo & 201,8 \\
\hline Thornthwait & 86,291 & 1,614 & 202,86 & $* *$ & 4,25 & 22,016 & 0,88 & 0,764 & 0,67 & Bom & 101,9 \\
\hline Camargo Simplificado & $\begin{array}{c}- \\
56,097\end{array}$ & 1,333 & 158,20 & $* *$ & 3,39 & 19,442 & 0,93 & 0,781 & 0,73 & Bom & 100,4 \\
\hline Camargo & $\begin{array}{c}- \\
65,669\end{array}$ & 1,379 & 204,79 & $* *$ & 3,94 & 22,121 & 0,98 & 0,740 & 0,73 & Bom & 96,1 \\
\hline Garcia Lopes & 5,279 & 1,028 & 43,19 & $* *$ & 1,40 & 10,158 & 0,92 & 0,901 & 0,83 & Muito Bom & 124,7 \\
\hline
\end{tabular}

* As médias são significativamente iguais ao nível de significância de 5\%

** As médias são significativamente diferentes ao nível de significância de 5\% 
Os resultados obtidos nesse período para a localidade de Linhares para a escala diária mostram que o método Garcia Lopez apresentou o melhor índice de desempenho $(c=0,63)$ caracterizado como mediano. Os demais métodos, Thornthwaite $(c=0,28)$, Camargo $(c=0,27)$ e Camargo Simplificado $(c=0,36)$, ficaram classificados com desempenho péssimo, enquanto que Priestley Taylor $(c=0,48)$ ficou classificado como mau.

Quanto à EEP o maior valor foi de Priestley Taylor $\left(3,529 \mathrm{~mm} \mathrm{dia}^{-1}\right)$ seguido pelo método de Camargo (1,366 mm dia $\left.{ }^{-1}\right)$, Camargo Simplificado $\left(1,201 \mathrm{~mm} \mathrm{dia}^{-1}\right)$, Thornthwaite $\left(0,944 \mathrm{~mm} \mathrm{dia}{ }^{-1}\right) \mathrm{e}$ Garcia Lopez (0,1 $\left.\mathrm{mm} \mathrm{dia}^{-1}\right)$. Deste sentido, o método de Priestley Taylor superestimou a ETo em 3,3 mm dia $^{-1}$ (80\%), o de Camargo subestimou em $1 \mathrm{~mm} \mathrm{dia}^{-1}$ (24\%), o de Camargo Simplificado 0,8 $\mathrm{mm} \mathrm{dia}^{-1}$ (19\%), Trornithwaite superestimando em $0,2 \mathrm{~mm} \mathrm{dia}^{-1}$ (4\%) e Garcia Lopes $0,1 \mathrm{~mm} \mathrm{dia}{ }^{-1}$ (2\%). Os resultados na escala diária demonstram que os métodos utilizados apresentam restrição ao uso para as condições climáticas do local de condução do trabalho, com menor restrição ao método de Garcia Lopez.

De acordo com Silva et al. (2011), os métodos de Thornthwaite e Camargo, na maioria das medidas, subestimaram tais valores, o mesmo observado por Araujo et al. (2007). Entretanto, no presente estudo apenas foi observado menores estimativas, ou melhor, subestimativas, pelo método de Camargo. Vescove; Turco (2005), também não obtiveram boa correlação desses métodos (Thornithwaite e Camargo) com o de Penman-Monteith para a região de Araraquara (SP), evidenciando o baixo ajuste para tais métodos.

Ao se analisar o EF (\%), observa-se que o método que apresentou o menor valor foi o de Garcia Lopez $(44,35 \%)$. Tal parâmetro encontrado explica que este método foi o mais que se aproximou do valor calculado, quando comparado ao padrão. Os demais métodos Priestley Taylor (1115,58\%), Camargo (167,29\%), Camargo Simplificado $(129,16 \%)$ e Thornthwaite $(79,83 \%)$ não obtiveram resultados satisfatórios.

Para Pereira et al. (2002), normalmente métodos empíricos como os de Thornthwaite e de Camargo, estimam bem a ETP na escala mensal, ao passo que métodos que envolvem o saldo de radiação apresentam boas estimativas também em escala diária.

O método de Garcia Lopez foi o que apresentou melhor resultado para a escala diária, sua classificação foi "mediana", semelhante ao encontrado por Fernandez e Gondim (1980) para a região de Areia, Paraíba.

$\mathrm{Na}$ escala de acumulado de três dias, todas as equações estudadas de Camargo, Camargo Simplificado e Garcia Lopez, apresentaram altos índices de precisão "r", de desempenho "c" e índice de concordância "d" mais próximo de "um", caracterizando maior concordância quando comparado à estimativa diária.

No que se refere à EEP para a escala de acumulado de três dias, o maior valor foi para Priestley Taylor (7,781 mm) seguido pelo método de Thornthwaite $(3,899 \mathrm{~mm})$, Camargo (2,125 mm), Camargo Simplificado $(2,117 \mathrm{~mm})$ e Garcia Lopez $(2,106 \mathrm{~mm})$. No tocante ao valor estimado, quando comparado ao método padrão, Priestley Taylor superestimou a ET0 em $7 \mathrm{~mm}$ (68\%), Thornthwaite 2,3 mm (26\%), Camargo subestimou em 1,1 mm (10\%), Camargo Simplificado em 1,1 mm (10\%) e por fim Garcia Lopez superestimou a ETP em $0,8 \mathrm{~mm}(7 \%)$. Neste sentido, o método que apresentou menor restrição para uso nas condições climáticas do local de condução do trabalho foi o método de Garcia Lopez.

Para o EF (\%) na escala de acumulado de três dias, verifica-se que o método que apresentou o menor valor foi novamente o de Garcia Lopez com 44,12\%. Isto permite induzir que este método aproximou melhor do valor estimado pelo método padrão. Já o método de Priestley Taylor $(602,36 \%)$, Thornthwaite (151,23\%), Camargo (44,94\%) e Camargo Simplificado (44,58\%), apresentaram resultados um pouco mais afastados do método padrão. Tal fato pode estar relacionado com a escala de tempo 
analisada, uma vez que os métodos citados anteriormente são mais recomendados para estimativa da evapotranspiração diária ou mensal, como é o caso do Thornthwaite (PEREIRA et al., 2002).

Nota-se pelos valores de "c" para a escala de três dias, que o método de Camargo Simplificado apresentou o melhor índice de desempenho $(c=0,70)$. Entretanto, não difere muito de Camargo $(c=0,69) \mathrm{e}$ Garcia Lopez $(\mathrm{c}=0,68)$, caracterizados também como "medianos". Para os métodos Thornthwaite $(\mathrm{c}=0,40)$ e Priestley Taylor (c=0,47), a classificação do desempenho ficou como "mau".

$\mathrm{Na}$ escala de acumulado de sete dias, os métodos de Priestley Taylor, Thornthwaite, Carmargo Simplificado e Camargo quando comparadas com o padrão Penman Monteith FAO (1998), apresentaram baixos índices de precisão "r", índices de desempenho "c" e índice de concordância "d", distantes de "um", caracterizando baixa concordância. Por outro lado, o método de Garcia Lopez apresentou melhor desempenho sendo considerado como "bom" com índices de precisão " $r$ " e de concordância " $d$ " mais próximo de um. Dessa forma, para o período de sete dias, somente o método de Garcia Lopez poderia ser recomendado na estimativa da ETo para as condições climáticas do local estudado.

Essa restrição pode ser detectada também em decorrência do elevado valor de EEP, os métodos Priestley Taylor (24,35 mm), seguido pelo método de Camargo (7,94 mm), Camargo Simplificado (7,34 $\mathrm{mm})$, Thornthwaite $(3,04 \mathrm{~mm})$ e Garcia Lopez $(2,73 \mathrm{~mm})$. Dentre os métodos mencionados, Priestley Taylor superestima a ETo em 23,7 mm (81\%), Camargo subestima em 6,8 mm (23\%), Camargo Simplificado em 5,7 mm (19\%), Thornthwaite em 1,2 mm (4\%) e o método de Garcia Lopez superestima em apenas $0,1 \mathrm{~mm}(0,3 \%)$, podendo, dessa forma, argumentar que o método que melhor estima a ETo na escala de sete dias é o de Garcia Lopez.

Ao analisar o EF (\%) para a escala de acumulado de sete dias, foi observado que o método que apresentou o menor valor foi Garcia Lopez com 51\%, seguido de Thornthwaite (63,08\%), Camargo Simplificado $(366,99)$, Camargo $(429,37 \%)$ e por fim Priestley Taylor $(4040,29 \%)$, isto indica que o método de Garcia Lopez, que teve o menor valor percentual, aproxima mais do valor estimado.

Com o valor obtido para o "c" verifica-se para a escala de sete dias que os métodos de Priestley Taylor ( $c=0,24)$, Camargo Simplificado $(c=0,36)$ e Camargo $(c=0,40)$ classificaram-se em "péssimo", enquanto que Thornthwaite ( $c=0,28)$ "sofrível" e Garcia Lopez $(c=0,71)$ caracterizado como "bom", apresentando, este último, melhor índice de desempenho.

$\mathrm{Na}$ escala de acumulado mensal, para os resultados obtidos, verifica-se que o método de Priestley Taylor alcançou baixo valor de precisão " $r$ ", índices de desempenho "c" e índice de concordância "d", enquanto que Thornthwaite, Carmargo Simplificado, Camargo e principalmente Garcia Lopez quando comparadas com o padrão Penman-Monteith FAO 1998, apresentaram valores mais altos de precisão "r", índices de desempenho "c" e índice de concordância "d" mais próximo de "um", caracterizando maior concordância. Para Oliveira et al. (2001) o fato deste último modelo empregar na sua formulação a umidade relativa, variável climática que mais influencia a evaporação, justifica a alta correlação dos valores medidos e estimados de ETo.

Quanto à EEP na análise dos dados de acumulado mensal, os maiores valores foram obtidos pelos métodos Priestley Taylor $(86,184 \mathrm{~mm})$, seguido pelo método de Camargo $(22,121 \mathrm{~mm})$, Thornthwaite (22,016 mm), Camargo Simplificado (19,442 mm) e Garcia Lopez (10,158 mm). Valores estimados por estes métodos superestimam a ETo em 85,6 mm (73\%) pelo Priestley Taylor, subestima em 20,1 mm (17\%) pelo Camargo, 14,3 mm (12\%) pelo Thornthwaite, 15,8 mm (13\%) pelo Camargo Simplificado e superestima em 8,5 mm (7\%) pelo método de Garcia Lopez. Dessa forma, pode-se esperar que o método que melhor estima a ETo na escala mensal, para a localidade em estudo, é o de Garcia Lopez, método este que diferentemente dos demais (Thornthwaite, Camargo Simplificado e Camargo), além de usar a temperatura para estimar a ETo, usa também a umidade relativa do ar, uma das principais 
variáveis responsáveis pela evapotranspiração e também utilizada no método padrão (GONÇALVES et al., 2009).

Para Conceição (2003), os métodos de estimativa de ETo que usam apenas a temperatura do ar como variável climática limitam a representatividade das condições climáticas para efeito de estimativas da evapotranspiração de referência porque conforme as condições de umidade e vento, a demanda hídrica atmosférica será diferente para os mesmos valores de temperatura do ar.

Detecta-se por meio da análise de EF (\%), para a escala de acumulado mensal, que o método que apresentou o valor mais próximo da unidade foi Garcia Lopez com 43,19\%, seguido de Camargo Simplificado (158,2\%), Thornthwaite $(202,86 \%)$, Camargo $(204,79 \%)$ e por fim Priestley Taylor (3108,67\%), significando que quanto menor o valor mais próximo do método de referencia se encontra.

Neste consenso, segundo os dados citados anteriormente, o método de Garcia Lopez foi o que mais adequou-se à região estudada para a escala de acumulado mensal. Resultados próximos aos observados foram encontrados por Stone e Silveira (1995) comparando nove modelos de estimativa de ETo com os resultados obtidos a partir da evaporação do tanque classe A para o município de Santo Antônio de Goiás. Entretanto, com base nos resultados obtidos os autores verificaram que o modelo de Penman foi o que apresentou maior coeficiente de correlação com os valores medidos pelo tanque classe A, seguido dos modelos de Hargreaves e Garcia-Lopez.

Para os índices de desempenho obtidos em "c" verifica-se para a escala mensal que os métodos de Priestley Taylor $(c=0,27)$ caracterizado como "Péssimo", Thornthwaite $(c=0,67)$, Camargo Simplificado e Camargo ( $c=0,73)$ classificaram-se em "Bom", enquanto que Garcia Lopez ( $c=083$ ) caracterizado como "Muito bom". Resultado semelhante foi encontrado por Fernadez e Gondim (1980) em Areia, PB quando estimava a ETo do local de estudo.

\section{CONCLUSÃO}

Para as condições climáticas registradas no período de janeiro a outubro de 2008 na localidade de Linhares, o método de estimativa de ETo que apresentou melhor resultado foi o de Garcia Lopez para as quatros escalas temporais estudadas.

Quando se dispõe apenas de dados de temperatura do ar, os resultados mostraram que pode-se usar o método de Camargo com boa confiabilidade para a localidade em estudo.

Não se recomenda o uso do método de Priestley Taylor para a localidade de Linhares para a estimativa de ETo em nenhuma das escala estudadas.

\section{REFERÊNCIAS}

ALENCAR, L. P. DE et al. Comparação de diferentes métodos de estimativa diária da evapotranspiração de referência para a região de Uberaba. Revista Brasileira de Ciências Agrárias, v. 6, n. 2, p. 337-343, abr./jun., 2011.

ALLEN, R.G.et al. Crop evapotranspiration: guidelines for computing crop water requirements. Rome: FAO, 1998. (FAO Irrigation and Drainage Paper, 56).

ALLEN, R. G. Penman for all seasons. Journal of Irrigation and Drainage Engineering, New York, v. 112, n. 4, p. 348-386, 1986. 
ARAÚJO, W. F; COSTA, S. A. A; SANTOS, A. E. Comparação entre métodos de estimativa da Evapotranspiração de referência (ETo) para Boa Vista, PR. Revista Caatinga, Mossoró, v.20, n.4, p.8488, jul./set. 2007.

CAMARGO, A. P; CAMARGO, M. B. P. Uma revisão analítica da evapotranspiração potencial. Bragantia, Campinas, v.59, n.2, p.125-137, 2000.

CAMARGO, A. P; SENTELHAS, P. C. Equação para a estimativa da evapotranspiração potencial baseada no método de Hargreaves - 1974. Revista Brasileira de agrometeorologia, Santa Maria, v. 4, n. 1, p. 77-81, 1996.

CONCEIÇÃO, M. A. F. Estimativa da evapotranspiração de referência com base na temperatura do ar para as condições do Baixo Rio Grande, SP. Revista Brasileira de Agrometeorologia, Santa Maria, v. 11, n. 2, p. 229-236, 2003.

DOORENBOS, J; PRUITT, W. O. Requerimentos de Água para as Culturas. Campina Grande-PB Universidade Federal da Paraíba (UFPB), jul.1997, 250 p.

FERNANDEZ, B; GONDIM, A. W. A. Estimativa de Evapotranspiração em Areia, PB. Revista Agropecuária Técnica, v. 1, n. 1, 1980.

GREENWOOD, D. J; NEETESON, J. J; DRAYCOTT, A response of potatoes to N fertilizer: dynamic model. Plant and soil. p. 185-203. 1985.

GONÇALVES, F. M. et al. Comparação de métodos da estimativa da evapotranspiração de referência para o município de Sobral-CE. Revista Brasileira de Agricultura Irrigada, v. 3, n. 2, p. 71-77, 2009.

NERY, J. T; CASTAÑEDA, E. Cálculo da evapotranspiração potencial na região de Maringá: estudo estatístico. Revista Unimar, Maringá, v. 14, n. 1, p. 59-74, 1992.

NÓBREGA, N. E. F. et al. Balanço hídrico climatológico e classificação climática de Thornthwaite e Köppen para o município de Linhares - ES. Linhares: Instituto Capixaba de Pesquisa, Assistência Técnica e Extensão Rural - Incaper. 2006. 6 p.

OLIVEIRA, G. M. DE. et al.. Comparação entre métodos de estimativa da evapotranspiração de referência na região norte da Bahia. Revista Brasileira de Agricultura Irrigada, v. 4, n. 2, p. 104-109, 2010.

OLIVEIRA, L. F. et al. Estudo comparativo de modelos de estimativa de evapotranspiração de referencia para algumas localidades no estado de Goiás e Distrito Federal. Revista Pesquisa Agropecuária Tropical, v. 31, n. 2, p. 121-126, 2001.

OLIVEIRA, R. A. et al. Desempenho do Irrigâmetro na estimativa da evapotranspiração de referência. Revista Brasileira de Engenharia Agrícola e Ambiental. v. 12, n. 2, p. 166-173, 2008.

PEREIRA, A. R; VILLA NOVA, N. A; SEDIYAMA, G. C. Evapo(transpi)ração. Piracicaba: FEALQ, 1997. 183p.

; ANGELOCCI, L. R; SENTELHAS, P. C. Agrometeorologia: Fundamentos e Aplicações Práticas. Guaíba: Agropecuária, 2002 478p.

PEREIRA, S. B. et al.. Evaporação líquida no lago de Sobradinho e impactos no escoamento devido à construção do reservatório. Revista Brasileira de Engenharia Agrícola e Ambiental. v. 13, n. 3, p.346$352,2009$.

REIS, L. F. C. et al. Estudo comparativo da estimativa da evapotranspiração de referencia para três localidades do Espírito Santo no período seco. Revista Idesia, Chile, v. 25, n. 3, p. 75-84, 2007. 
SCÁRDUA, J. A; FEITOZA, L. R; CASTRO, L. L. F. Estimativas da evapotranspiração potencial para o Estado do Espírito Santo. 2.ed. Vitória: Emcapa, 1986. 44 p. (Boletim de pesquisa, n. 6).

SILVA, V. J. et al. Desempenho de diferentes métodos de estimativa da evapotranspiração de referência diária em Uberlândia, MG. Revista BioScienci Journal, Uberlândia, v. 27, n. 1, p. 95-101, 2011.

SYPERREK, V. L. G. et al. Avaliação de desempenho de métodos para estimativas de evapotranspiração de referência para a região de Palotina, Estado do Paraná. Revista Acta Scienci Agronomic, Maringá, v. 30, n. 1, p. 603-609, 2008.

STONE, L. F.; SILVEIRA, P. M. 1995. Determinação da evapotranspiração para fins de irrigação. CNPAF - Embrapa. 49p. (Documento, 55).

VESCOVE, H. V; TURCO, J. E. P. Comparação de três métodos de estimativa da evapotranspiração de referência para a região de Araraquara - SP. Revista Brasileira de Engenharia Agrícola, Jaboticabal, v. 25, n. 3, p. 713-721, 2005.

WILLMOTT, C. J.; CKLESON, S. G; DAVIS, R. E. Statistics for evaluation and comparison of models. Journal of Geophysical Research, Ottawa, v. 90, n. C5. p. 8995-9005, 1985. 